\title{
Chemical and Biological Investigations on Mangrove Associated Lichens (Manglicolous Lichens) from India: A Review
}

\author{
V. B. TATIPAMULA, K. N. KILLARI ${ }^{1 *}$, HARITHA POLIMATI ${ }^{1}$ AND ALEKHYA KETHA ${ }^{1}$ \\ Institute of Research and Development, Duy Tan University, Da Nang 550000, Vietnam, ${ }^{1}$ Pharmaceutical Sciences \\ Department, AU College of Pharmaceutical Sciences, Andhra University, Visakhapatnam 530003, India
}

Tatipamula et al.: Studies on Manglicolous Lichens

\begin{abstract}
In 370-285 BC, an itinerant Greek philosopher Theophrastus phrased the term 'Lichen', which is a symbiosis between mycobiont (fungus) and a photobiont (algal) or a cyanobiont (cyanobacterium) where the mycobiont provides the shape and structure, while the photobiont or cyanobiont supply food, i.e., carbohydrates. From the folklore, lichen and its substances are used for various biological activities, which include antimicrobial, anticancer, anti-inflammatory, antioxidant and enzyme inhibitory activities. It is interesting to note that lichens are epiphytes as they synthesis their food by photosynthesis by using plants, rocks and dead logs as a substrate. In particular, lichens that betide to mangroves are termed as "Manglicolous Lichens". Generally, mangroves survive in a unique environment having a high concentration of salt and moisture, low and high tidal water. Hence, lichens growing in such stressful areas may lead to contain variation in their chemical constituents as well as pharmacological actions due to their defensive mechanism from the predators. Keeping the factors mentioned earlier, researchers worked and reported new lichen substances with potent biological properties from manglicolous lichens. The present review, list out all the chemical constituents and biological activities that are reported to date from manglicolous lichens in India. Also, this review remains useful for further research on manglicolous lichens to identify the potential bioactive molecules.
\end{abstract}

Key words: Mangroves, lichen substances, biological activity, manglicolous lichens, secondary metabolites

Mangroves are the only plants that exist in between land and sea waters. A unique ecosystem is built in and around mangroves with circumstances of anaerobic muddy soils, extreme tides, high temperatures and salinity, strong winds ${ }^{[1,2]}$. This unique ecosystem favors assemblage of many species, especially the muddy and sandy soil sediments attract bacteria, fungi, macroalgae and invertebrates and also the aerial roots and twigs host other groups of organisms ${ }^{[3-7]}$. Because of the adaptive characteristics, the ecology of mangroves has been extensively studied.

Usually, the harsh stressful environments of mangroves, for instance, variations in tidal water, also effects the epiphytes such as lichens that are habituated on them ${ }^{[8-12]}$. Lichen is a symbiotic organism, which is an association of fungi and algae and the lichens growing particularly on the mangroves are named as manglicolous lichens. Generally, the fungal matter present in lichens will die if they come in contact with

*Address for correspondence

E-mail: kishorenaidu.killari@gmail.com

March-April 2021 saltwater. Hence, it is a sporadic and unique case to find out lichens in mangrove forest regions. Moreover, lichens growing in such stressful areas may also lead to contain variation in their chemical constituents as well as pharmacological actions due to habitat under stress conditions and also their defensive mechanism from the predators $^{[12,13]}$.

Also, the identification of lichens is always misleading because of the symbiotic association of a microscopic algae or cyanobacterium with a filamentous fungus. Due to this, even they have full applications in human life, the studies carried out on them till now are minute ${ }^{[14,15]}$. Furthermore, it is substantial to study manglicolous

This is an open access article distributed under the terms of the Creative Commons Attribution-NonCommercial-ShareAlike 3.0 License, which allows others to remix, tweak, and build upon the work non-commercially, as long as the author is credited and the new creations are licensed under the identical terms

Accepted 03 March 2021

Revised 02 January 2021

Received 25 February 2020 Indian J Pharm Sci 2021;83(2):186-194 
lichens because of climatic factors in mangrove areas, fewer modes of transport and time lagging process due to damp soil, backwater flow and aerial roots (which adjourns the field survey). So a survey of especially manglicolous lichen is an important task ${ }^{[16]}$. As the lichens are generally termed as bio-monitors, they are very less resistant towards pollution. Even though there is less possibility of having pollution problems in and around mangrove regions, the lichens are profoundly affected by acid rains (which contain high amounts of sulfur dioxide). Eventually, acid rains affect the availability of lichens in mangroves ${ }^{[17-20]}$.

The literature on manglicolous lichens is very less. Long back, lichens on mangroves are included either within flora of a region or in monographic studies. Mangroves have rich and interesting lichen flora ${ }^{[21]}$. Initially, studies on epiphytic community growing on a mangrove tree Kandelia candel were performed which described several lichens and bryophytes, further ecology of mangrove lichens and environmental factors influencing pattern of lichen distribution in mangroves of Southeast Brazil were carried out, which leads to the updates of existing list of lichens of about 81 lichen genera from Southern Brazil and Itanhaem river mangroves. Similarly, unique lichens species of Peltigera from mangroves swamps of Colombian Caribbean coast were reported; this exemplifies the lichen richness in mangroves vegetation of tropical belt. Based on these, several studies were framed by several men like favorable growth factors of lichens on mangrove trees in inland streams to those growing near the river mouths and open seas ${ }^{[22-26]}$.

Though India has a large coastal area under mangrove vegetation under ten states and Union Territories, independent studies on lichens are rare in these areas, the studies of lichen flora in West Bengal state (a part of Sundarbans and Parmadan Forest) revealed 160 lichen taxa and proves the existence of diverse lichen flora in Sundarbans mangroves. Additionally, an in-deep survey on Sundarbans mangroves identified 550 specimens, which resulted in 165 species, of which 25 species were new records to India. Likewise, 13 and 12 lichens species were recorded from Muthupet lagoon and Pichavaram mangroves, respectively and some impressive records of Roccellaceae from the Gujarat mangroves were also recorded. Apart from these, there are no other reports of manglicolous lichens from other areas of India ${ }^{[26-28]}$.

Keeping all the drawbacks above and aspects, there are limited reports on the chemical and biological evaluation of particularly mangrove associated lichens. This review, list out all the chemical and biological investigations of associated mangrove lichens, till now. The list of manglicolous lichens that are examined were listed below.

Dirinaria consimilis (Stirton) D. D. Awasthi ${ }^{[29]}$, Host: twigs of Avicennia officinalis Location: Vainateya Island, Godavari estuary, West Godavari District, Andhra Pradesh, India.

Graphis ajarekarii Patw. \& C.R. Kulk ${ }^{[30]}$, Host: twigs of Avicennia officinalis, Location: Nagayalanka mangroves, Krishna estuary, Krishna District, Andhra Pradesh, India.

Parmotrema tinctorum (Despr. ex Nyl. ${ }^{[29]}$, Host: twigs of Excoecaria agallocha, Location: Bhitharkanika Island, Mahanadi estuary, Odisha, India.

Ramalina leiodea (Nyl.) Nyl. ${ }^{[30]}$, Host: twigs of Ceriops decandra, Location: Bhitharkanika Island, Mahanadi estuary, Odisha, India.

Roccella montagnei Bel em. D. D. Awasthi ${ }^{[29]}$, Host: twigs of Excoecaria agallocha, Location: Vainateya Island, Godavari estuary, West Godavari District, Andhra Pradesh, India.

\section{LICHEN SUBSTANCES ISOLATED FROM MANGLICOLOUS LICHENS}

The lichen substances that are isolated from the above species are represented in fig. 1 .

\section{2'-O-methyl divaricatic acid ${ }^{[31]}$ :}

$\mathrm{C}_{22} \mathrm{H}_{26} \mathrm{O}_{7}(402.44)$

White powder

Retention factor (Rf): 0.4 (1:1 ratio of hexane and ethyl acetate)

Wavelength of maximum absorbance $\left(\lambda_{\max }\right): 212.5 \mathrm{~nm}$ Melting point (Mp): 142-143

Elemental analysis: C-65.66, H-6.62 (\%)

Electrospray ionization-mass spectrometry (ESI-MS) $\mathrm{m} / \mathrm{z}: 403.05\left[\mathrm{M}+\mathrm{H}^{+}\right]$

Proton nuclear magnetic resonance ( $\left.{ }^{1} \mathrm{H} \quad \mathrm{NMR}\right)$ (Deuterated chloroform $\left.\left(\mathrm{CDCl}_{3}\right), 400 \mathrm{MHz}\right): 11.73$ (s, 1H), 11.18 (s, 1H), 6.46 (s, 1H), 6.40 (s, 2H), 3.91 $(\mathrm{s}, 3 \mathrm{H}), 3.85(\mathrm{~s}, 3 \mathrm{H}), 2.95-3.04(\mathrm{~m}, 4 \mathrm{H}), 1.66-1.80$ (m, 4H), 0.93-1.05 (m, 6H).

Carbon-13 nuclear magnetic resonance $\left({ }^{13} \mathrm{C}\right.$ NMR $)$ 


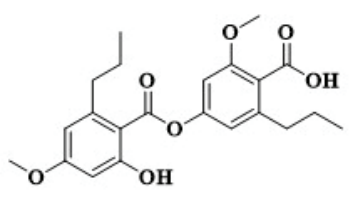

2'-O-Methyldivaricatic acid (1)
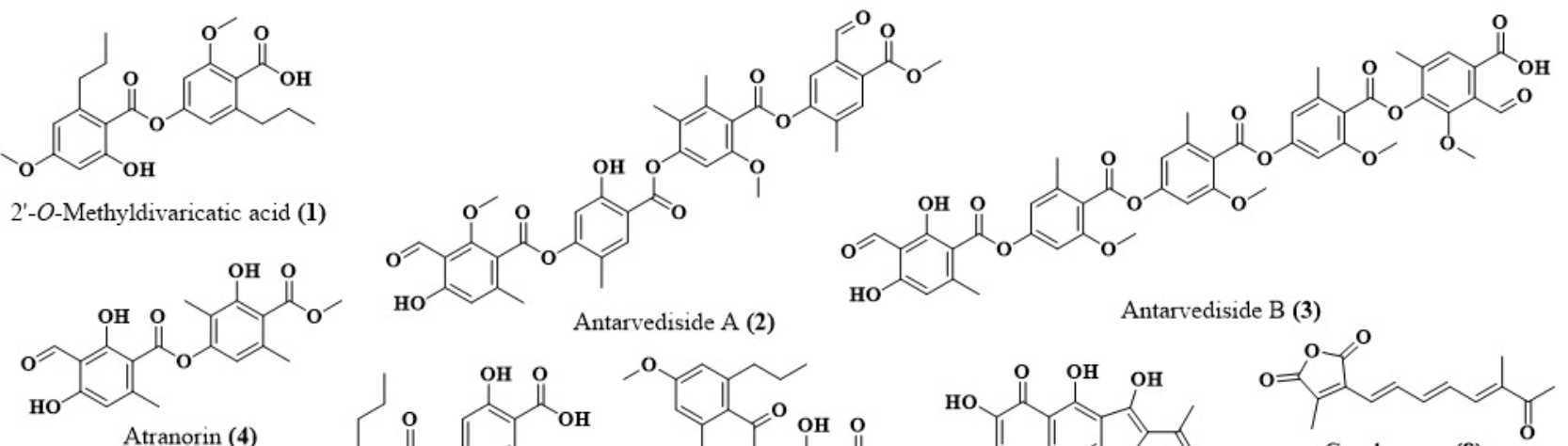

Antarvediside A (2)
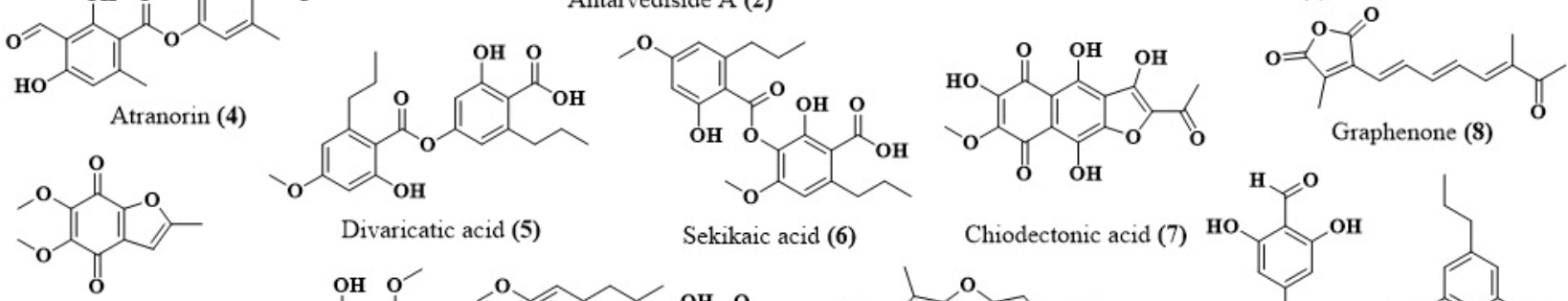

Graphisquinone (9)

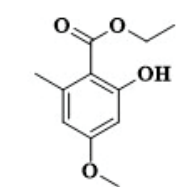

Ethyl everninate (10)
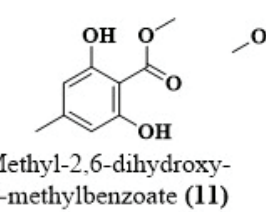

4-methylbenzoate (11)
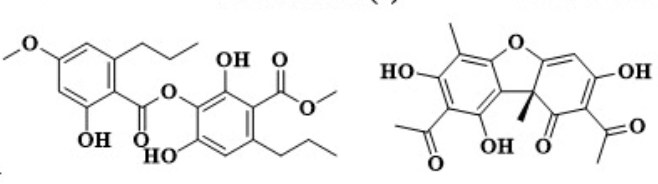

Scrobiculin (12)

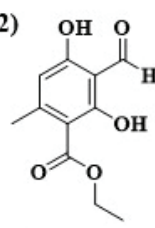

Usnic acid (13)

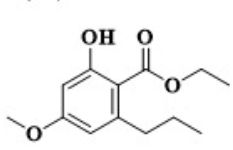

Ethyl divaricatinate (17)

Ethyl haematommate (18)

Divarinolmonomethylether (16)
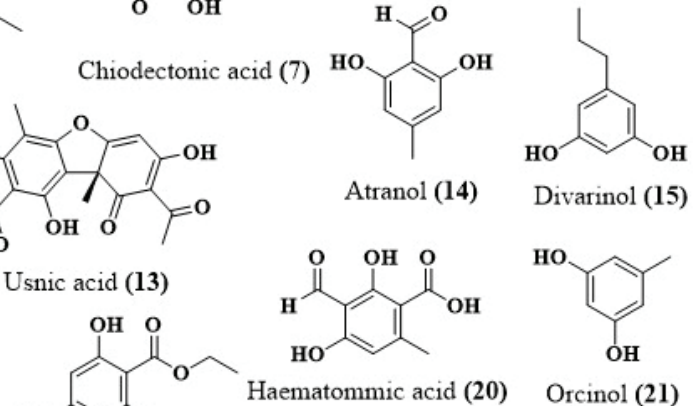

Atranol (14)

Divarinol (15)

HO

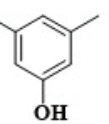

HO

Ethyl orsellinate (19)

Orcinol (21)

Fig. 1: Chemical structures of identified lichen substances from manglicolous lichens

$\left(\mathrm{CDCl}_{3}, 400 \mathrm{MHz}\right): 14.35,14.36,24.84,25.15,38.87$, $39.10,55.37,56.05,98.89,104.45,104.45,104.65$, $106.48,110.97,124.94,147.31,148.73,156.40,156.94$, $164.42,165.55,168.91$ and 175.47

\section{Antarvediside $\mathbf{A}^{[31]}$ :}

$\mathrm{C}_{38} \mathrm{H}_{34} \mathrm{O}_{14}(714.68)$

Pale pink needles

$\mathrm{R}_{\mathrm{f}}: 0.7$ (9:1 ratio of hexane and dichloromethane)

$\lambda_{\text {max }}: 222 \mathrm{~nm}$

Mp: $191-192^{\circ}$

Elemental analysis: C-63.76, H-4.82 (\%)

ESI-MS m/z: $715.00\left[\mathrm{M}+\mathrm{H}^{+}\right]$

${ }^{1} \mathrm{H}$ NMR $\left(\mathrm{CDCl}_{3}, 400 \mathrm{MHz}\right): 12.55$ (s, 1H), 12.50 (s, 1H), $11.95(\mathrm{~s}, 1 \mathrm{H}), 10.36(\mathrm{~s}, 1 \mathrm{H}), 6.52(\mathrm{~s}, 1 \mathrm{H}), 6.41(\mathrm{~s}$, 1H), 3.99 (s, 3H), 2.69 (s, 3H), $2.56(\mathrm{~s}, 3 \mathrm{H}), 2.18$ (s, $3 \mathrm{H}), 2.09(\mathrm{~s}, 3 \mathrm{H})$.

${ }^{13} \mathrm{C} \mathrm{NMR}\left(\mathrm{CDCl}_{3}, 400 \mathrm{MHz}\right): 9.39,23.46,24.05,25.60$, 29.73, 52.36, 55.67, 55.72, 92.08, 96.78, 98.47, 102.85, $104.13,108.56,110.28,110.53,112.87,112.96,115.46$, $116.03,116.80,139.89,143.47,152.00,152.45,156.95$, $159.42,162.89,163.74,163.77,165.82,167.50,169.10$, $169.71,172.22,182.41,193.86$.

\section{Antarvediside $B^{[31]}$ :}

$\mathrm{C}_{46} \mathrm{H}_{40} \mathrm{O}_{18}(880.81)$

White solid

$\mathrm{R}_{\mathrm{f}}: 0.2$ (7:3 ratio of hexane and ethyl acetate)

$\lambda_{\text {max }}: 219 \mathrm{~nm}$

Mp: 203-204

Elemental analysis: C-62.76, H-4.54 (\%)

ESI-MS m/z: $881.21\left[\mathrm{M}+\mathrm{H}^{+}\right]$

${ }^{1} \mathrm{H}$ NMR $\left(\mathrm{CDCl}_{3}, 400 \mathrm{MHz}\right): 13.39$ (s, 2H), 12.55 (s, $1 \mathrm{H}), 12.50(\mathrm{~s}, 1 \mathrm{H}), 11.95(\mathrm{~s}, 1 \mathrm{H}), 10.36(\mathrm{~s}, 1 \mathrm{H}), 6.67-$ 6.69 (dd, 3H, J=8 Hz), 6.52 (s, 1H), 6.40 (s, 1H), 6.306.34 (dd, 3H, J=12, $16 \mathrm{~Hz}), 3.99$ (s, 3H), 3.90 (s, 6H), $3.87(\mathrm{~s}, 3 \mathrm{H}), 2.85(\mathrm{~s}, 6 \mathrm{H}), 2.69(\mathrm{~s}, 3 \mathrm{H}), 2.55(\mathrm{~s}, 3 \mathrm{H})$, $2.09(\mathrm{~s}, 3 \mathrm{H})$.

${ }^{13} \mathrm{C} \mathrm{NMR}\left(\mathrm{CDCl}_{3}, 400 \mathrm{MHz}\right): 9.39,23.47,24.04,25.60$, 29.73, 52.36, 55.67, 55.73, 62.14, 92.10, 96.79, 98.49, 100.44, 108.57, 109.00, 112.88, 115.47, 115.56, 116.03, $119.36,143.50,149.52,152.46,153.44,162.90,163.76$, $164.17,167.51,168.72,182.44,185.78,193.87$.

\section{$\operatorname{Atranol}^{[32]}$ :}

$\mathrm{C}_{8} \mathrm{H}_{8} \mathrm{O}_{3}(152.15)$ 
Yellowish powder

$\mathrm{R}_{\mathrm{f}}: 0.8$ (7:3 ratio of dichloromethane and ethyl acetate)

$\lambda_{\max }: 225 \mathrm{~nm}$

Mp: $125-126^{\circ}$

Elemental analysis: C-63.50, H-5.52 (\%)

ESI-MS m/z: $153.10\left[\mathrm{M}+\mathrm{H}^{+}\right]$

${ }^{1} \mathrm{HNMR}\left(400 \mathrm{MHz}\right.$, dimethyl sulfoxide-d6(DMSO-d $\left.\mathrm{D}_{6}\right)$ ): 11.29 (s, 1H), 10.66 (s, 1H), 9.94 (s, 1H), 6.12 (s, 2H), $2.23(\mathrm{~s}, 3 \mathrm{H})$.

${ }^{13}$ C NMR (400 MHz, DMSO-d 6 ): 22.46, 100.83, 107.93, $110.58,141.15,161.46,192.87$.

\section{Atranorin $^{[31]}$ :}

$\mathrm{C}_{19} \mathrm{H}_{18} \mathrm{O}_{8}(374.35)$

Colorless needles

$\mathrm{R}_{\mathrm{f}}: 0.4$ (1:1 ratio of dichloromethane and ethyl acetate)

$\lambda_{\text {max }}: 210 \mathrm{~nm}$

Mp: $196-197^{\circ}$

Elemental analysis: C-60.76, H-4.74 (\%)

ESI-MS m/z: $375.11\left[\mathrm{M}+\mathrm{H}^{+}\right]$

${ }^{1} \mathrm{H}$ NMR ( $\left.\mathrm{CDCl}_{3}, 400 \mathrm{MHz}\right): 12.58$ (s, 1H), 12.52 (s, 1H), 11.97 (s, 1H), 10.39 (s, 1H), $6.54(\mathrm{~s}, 1 \mathrm{H}), 6.43$ (s, $1 \mathrm{H}), 4.01(\mathrm{~s}, 3 \mathrm{H}), 2.71(\mathrm{~s}, 3 \mathrm{H}), 2.57(\mathrm{~s}, 3 \mathrm{H}), 2.12(\mathrm{~s}$, $3 \mathrm{H})$.

${ }^{13} \mathrm{C} \mathrm{NMR}\left(\mathrm{CDCl}_{3}, 400 \mathrm{MHz}\right): 9.37,24.02,25.58,52.34$, $102.85,108.56,110.27,112.87,116.02,116.08,139.88$, $152.00,152.44,162.89,167.50,169.10,169.71,172.21$, 193.36 .

\section{Chiodectonic $\operatorname{acid}^{[33]}$ :}

$\mathrm{C}_{15} \mathrm{H}_{10} \mathrm{O}_{9}(334.24)$

Red color crystals

$\mathrm{R}_{\mathrm{f}}: 0.4$ (1:4 ratio of hexane and ethyl acetate)

$\lambda_{\max }: 287 \mathrm{~nm}$

Mp: 296-297

Elemental analysis: C-60.76, H-4.74 (\%)

ESI-MS m/z: $335.08\left[\mathrm{M}+\mathrm{H}^{+}\right]$

${ }^{1} \mathrm{H}$ NMR $\left(\mathrm{CDCl}_{3}, 400 \mathrm{MHz}\right): 5.84$ (s, 1H), 5.63 (s, 1H), $5.15(\mathrm{~s}, 1 \mathrm{H}), 3.79(\mathrm{~s}, 3 \mathrm{H}), 2.69(\mathrm{~s}, 3 \mathrm{H}), 1.41(\mathrm{~s}, 1 \mathrm{H})$.

${ }^{13} \mathrm{C}$ NMR $\left(\mathrm{CDCl}_{3}, 400 \mathrm{MHz}\right): 25.13,61.34,111.68$, 115.55, 118.60, 129.90, 145.69, 146.38, 148.59, 155.94,
$159.76,158.46,179.37,184.07,188.53$.

\section{Divaricatic acid ${ }^{[31]}$ :}

$\mathrm{C}_{21} \mathrm{H}_{24} \mathrm{O}_{7}(388.42)$

Pale pink solid

$\mathrm{R}_{\mathrm{f}}: 0.2$ (1:1 ratio of dichloromethane and ethyl acetate)

$\lambda_{\max }: 213 \mathrm{~nm}$

Mp: $138-139^{\circ}$

Elemental analysis: C-64.76, H-6.34 (\%)

ESI-MS m/z: $387.00\left[\mathrm{M}+\mathrm{H}^{+}\right]$

${ }^{1} \mathrm{H}$ NMR ( $\left.\mathrm{CDCl}_{3}, 400 \mathrm{MHz}\right): 11.36$ (s, 2H), 6.78 (s, $1 \mathrm{H}), 6.67(\mathrm{~s}, 1 \mathrm{H}), 6.40-6.42(\mathrm{~s}, 2 \mathrm{H}), 3.85(\mathrm{~s}, 3 \mathrm{H}), 2.96-$ $3.04(\mathrm{~m}, 4 \mathrm{H}), 1.70-1.72(\mathrm{~m}, 4 \mathrm{H}), 0.97-1.05(\mathrm{~m}, 6 \mathrm{H})$.

${ }^{13} \mathrm{C}$ NMR $\left(\mathrm{CDCl}_{3}, 400 \mathrm{MHz}\right): 14.19,14.23,24.83$, $25.28,38.54,39.18,55.43,99.03,103.58,108.50$, $108.99,111.59,116.27,148.11,149.62,154.98,164.89$, $165.35,166.61,169.39,174.67$.

\section{Divarinol $^{[32]}$ :}

$\mathrm{C}_{9} \mathrm{H}_{12} \mathrm{O}_{2}(152.19)$

Pinkish powder

$\mathrm{R}_{\mathrm{f}}: 0.8$ (1:1 ratio of hexane and ethyl acetate)

$\lambda_{\text {max }}: 274 \mathrm{~nm}$

Mp: $51-52^{\circ}$

Elemental analysis: C-71.87, H-7.82 (\%)

ESI-MS m/z: $153.0\left[\mathrm{M}+\mathrm{H}^{+}\right]$

${ }^{1} \mathrm{H}$ NMR (400 MHz, DMSO-d $)$ : 6.66 (s, 1H), 6.40-6.42 (dd, 1H, J=8, 9.2 Hz), 6.18 (s, 1H), 3.53 (brs, 2H), 2.95$3.04(\mathrm{~m}, 2 \mathrm{H}), 1.65-1.76(\mathrm{~m}, 2 \mathrm{H}), 0.97-1.04(\mathrm{~m}, 3 \mathrm{H})$.

${ }^{13} \mathrm{C}$ NMR (400 MHz, DMSO-d $): 12.40,23.11,35.21$, 101.49, 108.59, 141.81, 155.76.

\section{Divarinolmonomethylether ${ }^{[32]}$ :}

$\mathrm{C}_{10} \mathrm{H}_{14} \mathrm{O}_{2}(166.22)$

Pale yellow oil

$\mathrm{R}_{\mathrm{f}}: 0.6$ (9:1 ratio of hexane and dichloromethane)

$\lambda_{\max }: 273 \mathrm{~nm}$

ESI-MS m/z: $166.90\left[\mathrm{M}+\mathrm{H}^{+}\right]$

${ }^{1} \mathrm{H}$ NMR (400 MHz, DMSO-d $)$ : 6.61 (d, 1H, J=3 Hz), $6.49(\mathrm{~s}, 1 \mathrm{H}), 6.24(\mathrm{~d}, 1 \mathrm{H}, \mathrm{J}=2.1 \mathrm{~Hz}), 4.39$ (brs, 3H), 3.76 (brs, $1 \mathrm{H}), 2.66-2.74$ (m, 2H), 1.41-1.52 (m, 2H), $0.74-0.82(\mathrm{~m}, 3 \mathrm{H})$. 
${ }^{13} \mathrm{C}$ NMR (400 MHz, DMSO-d 6 ): 12.40, 23.11, 35.21, $52.81,101.49,108.59,111.23,141.81,155.76,162.12$.

\section{Ethyl divaricatinate $^{[32]}$ :}

$\mathrm{C}_{13} \mathrm{H}_{18} \mathrm{O}_{4}(238.28)$

Sharp colorless needles

$\mathrm{R}_{\mathrm{f}}: 0.4$ (9:1 ratio of hexane and ethyl acetate)

$\lambda_{\max }: 274 \mathrm{~nm}$

$\mathrm{Mp}: 51-52^{\circ}$

Elemental analysis: C-65.16, H-7.26 (\%)

ESI-MS m/z: $239.0\left[\mathrm{M}+\mathrm{H}^{+}\right]$

${ }^{1} \mathrm{H}$ NMR (400 MHz, DMSO-d 6 ): 11.36 (s, 1H), 6.79 (d, $1 \mathrm{H}, \mathrm{J}=2.8 \mathrm{~Hz}), 6.67(\mathrm{~d}, 1 \mathrm{H}, \mathrm{J}=2.3 \mathrm{~Hz}), 3.87(\mathrm{~s}, 3 \mathrm{H})$, 2.98-3.04 (m, 2H), 2.38-2.39 (m, 2H), 1.70-1.72 (m, $2 \mathrm{H}), 0.97-1.05(\mathrm{~m}, 6 \mathrm{H})$.

${ }^{13} \mathrm{C}$ NMR (400 MHz, DMSO-d $): 14.50,14.60,24.87$, $31.17,55.75,56.53,99.46,106.21,108.59,144.12$, $162.92,166.74,172.65$.

\section{Ethyl everninate ${ }^{[34]}$ :}

$\mathrm{C}_{11} \mathrm{H}_{14} \mathrm{O}_{4}(210.23)$

Greenish solid

$\mathrm{R}_{\mathrm{f}}: 0.4$ (9:1 ratio of hexane and dichloromethane)

$\lambda_{\text {max }}: 219 \mathrm{~nm}$

Mp: $74-76^{\circ}$

Elemental analysis: C-62.87; H-6.82 (\%)

ESI-MS m/z: $210.0\left[\mathrm{M}+\mathrm{H}^{+}\right]$

${ }^{1} \mathrm{H}$ NMR (400 MHz, Acetone-d $\left.\mathrm{d}_{6}\right) 6.37$ (d, 1H), 6.33 (d, $1 \mathrm{H}), 3.83(\mathrm{~s}, 3 \mathrm{H}), 2.09(\mathrm{~s}, 1 \mathrm{H}), 2.06(\mathrm{t}, 3 \mathrm{H}), 1.62(\mathrm{~m}$, $2 \mathrm{H}), 0.95(\mathrm{t}, 3 \mathrm{H})$.

${ }^{13} \mathrm{C}$ NMR (400 MHz, Acetone- $\mathrm{d}_{6}$ ): 14.47, 25.85, 54.96, 55.80, 99.70, 111.10, 149.10, 165.20, 167.20, 174.00.

\section{Ethyl haematommate ${ }^{[32]}$ :}

$\mathrm{C}_{11} \mathrm{H}_{12} \mathrm{O}_{5}(224.21)$

Greenish solid

$\mathrm{R}_{\mathrm{f}}: 0.6$ (7:3 ratio of dichloromethane and ethyl acetate)

$\mathrm{Mp}: 112-113^{\circ}$

$\lambda_{\max }: 209.5 \mathrm{~nm}$

Elemental analysis: C-58.64, H-5.52 (\%)

ESI-MS m/z: $224.90\left[\mathrm{M}+\mathrm{H}^{+}\right]$
${ }^{1} \mathrm{H}$ NMR (400 MHz, DMSO-d $\left.{ }_{6}\right) 13.75$ (s, 1H), 11.46 (s, 1H), 10.59 (s, 1H), $9.68(\mathrm{~s}, 1 \mathrm{H}), 6.42(\mathrm{~s}, 1 \mathrm{H}), 2.54$ (s, 3H), 1.59-1.65 (m, 2H), 0.93-0.97 (t, 3H).

${ }^{13} \mathrm{C}$ NMR (400 MHz, DMSO-d $)$ : 14.28, 20.14, 68.63, $106.38,109.34,114.78,155.22,163.90,167.04,173.42$, 191.73.

\section{Ethyl orsellinate ${ }^{[32]}$ :}

$\mathrm{C}_{10} \mathrm{H}_{12} \mathrm{O}_{4}(196.20)$

Pale yellow solid

$\mathrm{R}_{\mathrm{f}}: 0.4$ (7:3 ratio of dichloromethane and ethyl acetate)

$\lambda_{\text {max }}: 219 \mathrm{~nm}$

Mp: $131-132^{\circ}$

Elemental analysis: C-61.26, H-6.55 (\%)

ESI-MS m/z: $197.10\left[\mathrm{M}+\mathrm{H}^{+}\right]$

${ }^{1} \mathrm{H}$ NMR (400 MHz, DMSO-d $)$ ): 10.59 (s, 1H), 9.68 $(\mathrm{s}, 1 \mathrm{H}), 6.42(\mathrm{~s}, 2 \mathrm{H}), 2.54(\mathrm{~s}, 3 \mathrm{H}), 1.46-1.52(\mathrm{~m}, 2 \mathrm{H})$, 0.80-0.84 (m, 3H).

${ }^{13} \mathrm{C}$ NMR (400 MHz, DMSO-d $)$ : 14.28, 20.14, 68.63, $105.25,109.34,114.78,155.22,163.90,173.42$.

\section{Graphenone $^{[33]}$ :}

$\mathrm{C}_{14} \mathrm{H}_{14} \mathrm{O}_{4}(246.26)$

Yellowish needles

$\mathrm{R}_{\mathrm{f}}: 0.6$ (1:1 ratio of hexane and ethyl acetate)

$\lambda_{\max }: 290 \mathrm{~nm}$

Mp: $167-168^{\circ}$

Elemental analysis: C-68.71, H-5.72 (\%)

ESI-MS m/z: $247.18\left[\mathrm{M}+\mathrm{H}^{+}\right]$

${ }^{1} \mathrm{H}$ NMR $\left(\mathrm{CDCl}_{3}, 400 \mathrm{MHz}\right): 7.05$ (s, 1H), 6.43-6.53 (m, 5H), $2.34(\mathrm{~s}, 3 \mathrm{H}), 2.12(\mathrm{~s}, 3 \mathrm{H}), 1.93(\mathrm{~s}, 3 \mathrm{H})$.

${ }^{13} \mathrm{C}$ NMR $\left(\mathrm{CDCl}_{3}, 400 \mathrm{MHz}\right): 12.34,12.61,27.54$, $125.00,128.70,129.02,131.40,131.97,132.65$, $134.73,143.30,166.28,168.61,200.83$.

\section{Graphisquinone $^{[33]}$ :}

$\mathrm{C}_{11} \mathrm{H}_{10} \mathrm{O}_{5}(222.20)$

Red needles

$\mathrm{R}_{\mathrm{f}}: 0.4$ (1:1 ratio of hexane and ethyl acetate)

$\lambda_{\text {max }}: 228 \mathrm{~nm}$

Mp: $130-131^{\circ}$ 
Elemental analysis: C-59.12, H-4.02 (\%)

ESI-MS m/z: $223.21\left[\mathrm{M}+\mathrm{H}^{+}\right]$

${ }^{1} \mathrm{H} \mathrm{NMR}\left(\mathrm{CDCl}_{3}, 400 \mathrm{MHz}\right): 7.17$ (s, 1H), 3.75 (s, 3H), $3.71(\mathrm{~s}, 3 \mathrm{H}), 2.38(\mathrm{~s}, 3 \mathrm{H})$.

${ }^{13} \mathrm{C} \mathrm{NMR}\left(\mathrm{CDCl}_{3}, 400 \mathrm{MHz}\right): 14.37,61.33,104.36$, 118.91, 144.94, 150.85, 153.57, 154.24, 176.98, 183.80 .

Haematommic acid $^{[32]}$ :

$\mathrm{C}_{9} \mathrm{H}_{8} \mathrm{O}_{5}(196.16)$

Pale yellow needles

$\mathrm{R}_{\mathrm{f}}: 0.4$ (1:1 ratio of hexane and ethyl acetate)

$\lambda_{\max }: 219.5 \mathrm{~nm}$

Mp: $172-173^{\circ}$

Elemental analysis: C-55.64, H-4.52 (\%)

ESI-MS m/z: $198.30\left[\mathrm{M}+\mathrm{H}^{+}\right]$

${ }^{1} \mathrm{H}$ NMR (400 MHz, DMSO-d $\left.{ }_{6}\right): 13.75$ (s, 1H), 11.46

(s, 1H), 10.59 (s, 1H), $9.68(\mathrm{~s}, 1 \mathrm{H}), 6.42(\mathrm{~s}, 1 \mathrm{H}), 2.54$ (s, 3H).

${ }^{13} \mathrm{C}$ NMR (400 MHz, DMSO-d 6 ): 17.10, 105.25, 109.34, 155.22, 163.90, 167.04, 173.42, 191.73 .

Methyl 2,6-dihydroxy-4-methylbenzoate ${ }^{[32]}$ :

$\mathrm{C}_{9} \mathrm{H}_{10} \mathrm{O}_{4}(182.18)$

Faint yellow crystals

$\mathrm{R}_{\mathrm{f}}: 0.6$ (1:1 ratio of hexane and ethyl acetate)

$\lambda_{\max }: 219.5 \mathrm{~nm}$

Mp: $143-144^{\circ}$

Elemental analysis: C-59.36; H-5.52 (\%)

ESI-MS m/z: $182.0\left[\mathrm{M}+\mathrm{H}^{+}\right]$

${ }^{1} \mathrm{H}$ NMR (400 MHz, DMSO-d ) $) 10.65$ (s, 1H), 9.94 (s, 1H), $6.12(\mathrm{~d}, 2 \mathrm{H}), 3.75(\mathrm{~s}, 3 \mathrm{H}), 2.23(\mathrm{~s}, 3 \mathrm{H})$.

${ }^{13} \mathrm{C}$ NMR (400 MHz, DMSO-d $)$ : 22.5, 52.2, 100.8, 107.9, 110.6, 141.2, 161.5, 170.6.

Orcinol $^{[32]}$ :

$\mathrm{C}_{7} \mathrm{H}_{8} \mathrm{O}_{2}$ (124.14)

Colorless needles

$\mathrm{R}_{\mathrm{f}}: 0.7$ (1:1 ratio of hexane and ethyl acetate)

$\lambda_{\max }: 215.5 \mathrm{~nm}$

Mp: $108-109^{\circ}$

Elemental analysis: C-67.59, H-6.52 (\%)
ESI-MS m/z: $125.10\left[\mathrm{M}+\mathrm{H}^{+}\right]$

${ }^{1} \mathrm{H}$ NMR (400 MHz, DMSO-d $)$ ): 6.30 (s, 1H), 6.23 (s, $2 \mathrm{H}), 3.34$ (s, 2H), 2.46 (s, 3H).

${ }^{13} \mathrm{C}$ NMR (400 MHz, DMSO-d $)$ ): 27.93, 98.36, 109.34, $155.22,163.90,157.52$.

\section{Scrobiculin $^{[34]}$ :}

$\mathrm{C}_{22} \mathrm{H}_{26} \mathrm{O}_{8}(418.44)$

Colorless crystals

$\mathrm{R}_{\mathrm{f}}: 0.6$ (7:3 ratio of dichloromethane and ethyl acetate)

$\lambda_{\max }: 217.5 \mathrm{~nm}$

Mp: $134-136^{\circ}$

Elemental analysis: C-63.16; H-6.26 (\%)

ESI-MS m/z: $418.0\left[\mathrm{M}+\mathrm{H}^{+}\right]$

${ }^{1} \mathrm{H}$ NMR (400 MHz, DMSO-d ${ }_{6}$ ): 11.85 (s, 1H), 10.50 (s, 1H), 6.61 (s, 1H), 6.39-6.41 (dd, 2H), 3.84 (s, 3H), $3.78(\mathrm{~s}, 3 \mathrm{H}), 2.82-2.87(\mathrm{dd}, 4 \mathrm{H}), 2.51(\mathrm{t}, 1 \mathrm{H}), 1.59-1.65$ (dd, 4H), 0.88-0.97 (m, 6H).

${ }^{13} \mathrm{C}$ NMR (400 MHz, DMSO-d $)$ : 14.5, 14.6, 24.8, 25.2, 36.9, 37.8, 55.8, 56.5, 99.5, 106.2, 108.6, 108.8, 109.5, $125.2,144.1,146.0,154.3,154.7,160.6,162.9,166.7$, 172.7 .

\section{Sekikaic acid $^{[31]}$ :}

$\mathrm{C}_{22} \mathrm{H}_{26} \mathrm{O}_{8}(418.44)$

White solid

$\mathrm{R}_{\mathrm{f}}: 0.6$ (1:1 ratio of dichloromethane and ethyl acetate)

$\lambda_{\text {max }}: 219 \mathrm{~nm}$

Mp: $220-221^{\circ}$

Elemental analysis: C-63.16, H-6.24 (\%)

ESI-MS m/z: $419.33\left[\mathrm{M}+\mathrm{H}^{+}\right]$

${ }^{1} \mathrm{H}$ NMR $\left(\mathrm{CDCl}_{3}, 400 \mathrm{MHz}\right): 11.40$ (s, 2H), 10.39 (s, $1 \mathrm{H}), 6.77-6.78$ (d, J=3.8 Hz, 1H), 6.66-6.67 (d, J=3.2 $\mathrm{Hz}, 1 \mathrm{H}), 6.40-6.42$ (dd, J=4, $8 \mathrm{~Hz}, 2 \mathrm{H}), 3.91$ (s, 3H), $3.85(\mathrm{~s}, 3 \mathrm{H}), 2.95-3.04(\mathrm{~m}, 4 \mathrm{H}), 1.69-1.72(\mathrm{~m}, 4 \mathrm{H})$, 0.97-1.05 (m, 6H).

${ }^{13} \mathrm{C} \mathrm{NMR}\left(\mathrm{CDCl}_{3}, 400 \mathrm{MHz}\right): 174.34,169.40,166.60$, 165.33, 164.88, 154.92, 149.53, 148.11, 116.22, 111.58, $108.55,103.60,99.03,55.43,39.18,38.53,25.28$, $24.84,14.23,14.18$.

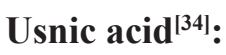

$\mathrm{C}_{18} \mathrm{H}_{16} \mathrm{O}_{7}(344.32)$ 


\begin{tabular}{|c|c|}
\hline Manglicolous lichen & Isolated Metabolites \\
\hline & $\begin{array}{c}\text { 2'-0-methyl divaricatic acid (1) } \\
\text { Antarvediside A (2) }\end{array}$ \\
\hline Dirinaria consimilis (Stirton) D. D. & Antarvediside B (3) \\
\hline \multirow[t]{3}{*}{ Awasthi ${ }^{[31]}$} & Atranorin (4) \\
\hline & Divaricatic acid (5) \\
\hline & Sekikaic acid (6) \\
\hline & Atranorin (4) \\
\hline Graphis ajarekarii Patw. \& C.R. & Chiodectonic acid (7) \\
\hline \multirow{2}{*}{ Kulk $^{[33,35,40]}$} & Graphenone (8) \\
\hline & Graphisquinone (9) \\
\hline \multirow[t]{3}{*}{ Parmotrema tinctorum (Despr. ex Nyl.. ${ }^{[35]}$} & Ethyl everninate (10) \\
\hline & 2'-O-methyl divaricatic acid (1) \\
\hline & Ethyl everninate $(10)$ \\
\hline \multirow[t]{6}{*}{ Ramalina leiodea (Nyl.) Nyl. ${ }^{34]}$} & Methyl-2,6-dihydroxy-4-methyl benzoate (11) \\
\hline & Scrobiculin (12) \\
\hline & Usnic acid (13) \\
\hline & Atranol (14) \\
\hline & Divarinol (15) \\
\hline & Divarinolmonomethylether (16) \\
\hline \multirow{6}{*}{$\begin{array}{l}\text { Roccella montagnei Bel em. D.D. } \\
\text { Awasthi[3 }^{32]}\end{array}$} & Ethyl divaricatinate (17) \\
\hline & Ethyl haematommate (18) \\
\hline & Ethyl orsellinate (19) \\
\hline & Haematommic acid (20) \\
\hline & Methyl-2,6-dihydroxy-4-methyl benzoate (11) \\
\hline & Orcinol (21) \\
\hline
\end{tabular}

Yellowish needles

$\mathrm{R}_{\mathrm{f}}: 0.4$ (9:1 ratio of hexane and dichloromethane)

$\lambda_{\text {max }}: 220 \mathrm{~nm}$

Mp: $201-202^{\circ}$

Elemental analysis: C-63.16; H-6.26 (\%)

ESI-MS m/z: $345.00\left[\mathrm{M}+\mathrm{H}^{+}\right]$

${ }^{1} \mathrm{H}$ NMR (400 MHz, $\left.\mathrm{CDCl}_{3}\right): 13.31(\mathrm{~s}, 1 \mathrm{H}), 11.03$ (s, 1H), $5.98(\mathrm{~s}, 1 \mathrm{H}), 2.68(\mathrm{~s}, 3 \mathrm{H}), 2.67(\mathrm{~s}, 3 \mathrm{H}), 2.11$ (s, 3H), $1.76(\mathrm{~s}, 3 \mathrm{H})$.

${ }^{13} \mathrm{C}$ NMR (400 MHz, $\mathrm{CDCl}_{3}$ ): 7.6, 27.9, 31.3, 32.2, 59.1, 98.4, 101.6, 104.0, 105.3, 109.3, 155.2, 157.5, $163.9,179.4,191.7,198.1,200.4,201.8$.

\section{BIOLOGICAL EXAMINATION OF EXTRACTS AND SECONDARY METABO- LITES OF MANGLICOLOUS LICHENS}

Extracts of manglicolous lichens showed different biological properties, including antioxidant, antibacterial, anti-cancer, antifungal, anti-inflammatory, anti-tubercular, hypo and hyperglycemic activities ${ }^{[35-39]}$.

Ethyl acetate and acetone extracts of manglicolous lichen Dirinaria consimilis showed a reasonable specificity against human lung adenocarcinoma cell line (A549), human colon cancer cell line (DLD-1), a hypopharyngeal carcinoma cell line (FADU), immortal cell line (HeLa) and breast cancer cell line (MCF-7) ${ }^{[38,39]}$. Also, the acetone extract of manglicolous lichen Dirinaria consimilis revealed prominent inhibition of protein denaturation, as well as inflammation in rat paw edema ${ }^{[39]}$. Ethanolic extract of manglicolous lichen Graphis ajarekarii showed fibrinolytic (in vitro) and anti-inflammatory activities (in vitro and in vivo) and a better specificity against human prostate cancer cell line (DU145), FADU, leukemia cell line (K-562), MCF7, epithelial, human breast cancer cell line (MDAMB-231) and human colon cancer cell line (SW620) ${ }^{[33,40]}$. Additionally, ethyl acetate extract of mangrove associated lichen Graphis ajarekarii is active against Bacillus subtilis, Micrococcus luteus, Salmonella Typhi, Candida albicans and Mycobacterium tuberculosis, free radicals (2,2-diphenyl-1-picrylhydrazyl (DPPH), (2,2'-azino-bis(3-ethylbenzothiazoline-6-sulfonic acid)) (ABTS) and superoxide) and also showed prominent hypo and hyperglycemic activities in albino $\operatorname{rats}^{[35,36]}$.

Manglicolous lichen Parmotrema tinctorum ethyl acetate extract is active against $S$. Typhi, free radicals (DPPH, ABTS and superoxide) and also showed prominent hypo and hyperglycemic activities in albino rats. Acetone extract of manglicolous lichen P. tinctorum is active against $B$. substilis and M. luteus $^{[35,36]}$. Ethyl acetate and acetone extract of mangrove associated lichen Ramalina leiodea showed functional inhibition 
of protein denaturation and a better specificity against A549, DLD-1, FADU, HeLa and MCF- ${ }^{738]}$. Chloroform and methanol extracts of mangrove associated lichen Roccella montagnei were active against S. aureus, DLD-1 and MCF-7. Ethyl acetate extract of manglicolous lichen $R$. montagnei is active against $B$. substilis. Acetone extract of mangrove associated lichen $R$. montagnei showed functional inhibition of free radicals (DPPH, ABTS and superoxide) and a significant specificity against A549, DLD-1, FADU and $\mathrm{HeLa}^{[37]}$. List of secondary metabolites identified from manglicolous lichens is shown in Table 1.

It was reported that 1 and 3 were active against DPPH, ABTS and superoxide free radicals and pronounced specificity against A549, DLD-1, FADU, HeLa and MCF-7, while 2, 11, 16 and 18 were active against ABTS, DPPH and superoxide free radicals. 4, 18 and 20 were active against protein denaturation, whereas 5 and 6 reported having a noticeable specificity against DLD-1, FADU and HeLa. 7, 8, 9, 13, 15, 20 and 21 were active against DPPH and superoxide free radicals, while 7 and 9 reported having a prominent specificity against FADU, MDA-MB-231 and SW620. 10 was active against superoxide free radicals only and 12 was reported to have a distinct specificity against A549, DLD-1, FADU, HeLa and MCF-7. 14 was reported to be active against ABTS and superoxide free radicals. 17 was reported to have a noticeable specificity against MCF-7 and HeLa, while 18 and 20 reported having a prominent specificity against A549, FADU and HeLa. 19 and 20 were reported to have moderate active against Mycobacterium tuberculosis H37Rv strain, while 19 reported having a marked specificity against DLD-1 ${ }^{[31-35,40]}$.

\section{CONCLUSION}

The chemical and biological investigation on lichens has exploited that they contain highly oxygenated aromatic derivatives with a wide range of biological activities. Also, more than 1000 secondary metabolites have been identified and reported from different lichen species, till now. However, there are no studies related to chemical and pharmacological examination on stressed lichens like manglicolous lichens. In many cases, it is proved that stressed organisms have a chance of obtaining novel compounds with potent biological activities.

Similarly, manglicolous lichens (stressed under mangrove ecosystems) are proved to have unique lichen substances with prominent pharmacological activities, which were listed out in this review. Moreover, as there are very few isolations and biological evaluation reports on manglicolous lichens, there is still a need to carry out extensive studies on manglicolous lichens, to discover unexplored substances of lichens along with their pharmacological evaluation. Hence, this is a first review report on the chemical and pharmacological evaluation of manglicolous lichens, which assist academic researchers and pharmaceutical scientists in working not only on manglicolous lichens but also on stressed organisms.

\section{Conflicts of Interest:}

The authors declared no conflict of interest.

\section{REFERENCES}

1. Hogarth PJ. The biology of mangroves and seagrasses. $3^{\text {rd }}$ ed. Oxford University Press; 2015.

2. Alongi D. The energetics of mangrove forests. Springer Science \& Business Media; 2009.

3. Tomlinson PB. The botany of mangroves. $2^{\text {nd }}$ ed. Cambridge University Press; 2016.

4. Das SK, Patra JK, Thatoi H. Antioxidative response to abiotic and biotic stresses in mangrove plants: A review. Int Rev Hydrobiol 2016;101:3-19.

5. Duke NC. Australia's mangroves: the authoritative guide to Australia's mangrove plants. MER; 2006.

6. Aksomkoae S. Ecology and management of mangroves. Bangkok, Thailand: IUCN-The World Conservation Union; 1993.

7. Boto KG, Wellington JT. Soil characteristics and nutrient status in a northern Australian mangrove forest. Estuaries 1984;7(1):61-9.

8. Blasco F, Saenger P, Janodet E. Mangroves as indicators of coastal change. Catena 1996;27:167-78.

9. Davenport J, Black KD, Burnell G, Cross T, Culloty S, Ekaratne S, et al. Aquaculture: the ecological issues. John Wiley \& Sons; 2009.

10. Kathiresan K, Bingham BL. Biology of mangroves and mangrove ecosystems. Adv Mar Biol 2001;40:84-254.

11. Ng PK, Corlett R, Tan HT, editors. Singapore biodiversity: an encyclopedia of the natural environment and sustainable development. Editions Didier Millet; 2011.

12. Ahmadjian V. The lichen symbiosis. John Wiley \& Sons; 1993.

13. Faeth SH. Are endophytic fungi defensive plant mutualists? Oikos 2002;98(1):25-36.

14. Brodo IM, Sharnoff SD, Sharnoff S. Lichens of North America. Yale University Press; 2001.

15. DePriest PT. Early molecular investigations of lichen-forming symbionts: 1986-2001. Annu Rev Microbiol 2004;58:273-301.

16. Clough B. Continuing the journey amongst mangroves. ISME mangrove educational book series; 2013. p. 86.

17. Jagtap TG, Nagle VL. Response and adaptability of mangrove habitats from the Indian subcontinent to changing climate. Ambio 2007;36(4):328-34.

18. Sett R, Kundu M. Epiphytic lichens: their usefulness as bio-indicators of air pollution. Donnish J Res Environ Stud 2016;3(3):17-24.

19. Shukla V, Upreti DK, Bajpai R. Lichen diversity in different lichenogeographical regions of India. In Lichens to Biomonitor 
the Environment. New Delhi: Springer; 2014. p. 61-96.

20. Lucban MC, Paguirigan JA. Occurrence of manglicolous lichens in Calabarzon, Philippines. Stud Fungi 2019;4(1):26373.

21. Mitra B, Biswas O, Roy S, Chakraborti U. Pollinators of mangroves in the perspective of Indian Sunderbans. ENVIS Newsl 2015;21:6-11.

22. Nayaka S, Ingle KK, Bajpai R, Rawal JR, Upreti DK, Trivedi S. Lichens of Gujarat state, India with special reference to coastal habitats. Curr Res Environ Appl Mycol 2013;3(2):2229.

23. Avendano-Remolina D, Sanjuan-Munoz A, Alvarez-Leon $\mathrm{R}$. New lichen in the mangrove swamps of the Colombian Caribbean coast. Rev Biol Trop 2000;48:719-24.

24. Chandy S, Euler DL. Can community forestry conserve tigers in India? In Personal, societal and ecological values of wilderness: Sixth World Wilderness Congress proceedings on research, management and allocation 1998;2:155-161.

25. Nayaka S, Upreti DK, Ingle KK. Mangroves of India: Potential habitats for unique lichen flora. Proceedings on International Day for Biological Diversity-Marine Biodiversity 2012:68-74.

26. Manjunath KR, Kumar $\mathrm{T}$, Kundu N, Panigrahy $\mathrm{S}$. Discrimination of mangrove species and mudflat classes using in situ hyperspectral data: a case study of Indian Sundarbans. GIsci Remote Sens 2013;50(4):400-17.

27. Logesh AR, Upreti DK, Kalaiselvam M, Nayaka S, Kathiresan K. Lichen flora of Pichavaram and Muthupet mangroves (Southeast coast of India). Mycosphere 2012;3:884-8.

28. Logesh AR, Kalaiselvam M, Upreti DK, Nayaka S, Kathiresan K. Mangroves-An abode for unique lichens. Coastal ecosystems of India, special publication. Annamalai University: Parangipettai; 2013. p. 39-44.

29. Bharadwaj VT, Sastry GV, Murthy KS. A note on the occurrence of lichens on Vainateya Godavari mangroves in East Godavari district of Andhra Pradesh India. Stud Fungi 2018;3(1):302-8.

30. Bharadwaj V. New record of mangrove lichens from Andhra Pradesh and Orissa states of India. Stud Fungi 2019;4(1):90-3.

31. Tatipamula VB, Vedula GS, Sastry AV. Antarvediside AB from manglicolous lichen Dirinaria consimilis (Stirton) DD
Awasthi and their pharmacological profile. Asian $\mathrm{J}$ Chem 2019;31(4):805-12.

32. Tatipamula VB, Vedula GS, Sastry AV. Chemical and pharmacological evaluation of manglicolous lichen Roccella montagnei Bel em. DD Awasthi. Futur J Pharm Sci 2019;5(1):19.

33. Haritha P, Patnaik SK, Tatipamula VB. Chemical and Pharmacological Evaluation of manglicolous lichen Graphis Ajarekarii Patw. \& Cr Kulk. Vietnam J Sci Technol 2019;57(3):300-8.

34. Tatipamula VB, Vedula GS. Antimicrobial and anti-tubercular activities of isolates and semi synthetic derivatives of lichen Ramalina leiodea (Nyl.) Nyl. J Serb Chem Soc 2019;84(6):55562.

35. Tatipamula VB, Vedula GS, Rathod BB, Shetty PR, Sastry AV. Study of phytochemical analysis, total flavonoid and phenolic content, antimicrobial properties and chemical constituents of two manglicolous lichens extracts. Inventi Rapid: Planta Activa 2018;2018(2):1-6.

36. Tatipamula VB, Vedula GS, Paidi KR, Annam SS. Nutraceutical value of lichens, Graphis ajarekarii and Parmotrema tinctorum and their implications in diabetes. Inventi Impact: Nutraceuticals 2018;2018(3):189-94.

37. Sastry AV, Vedula GS, Tatipamula VB. In vitro biological profile of mangrove associated lichen, Roccella montagnei extracts. Inventi Impact: Ethnopharmacol 2018;2018(3):1538 .

38. Tatipamula VB, Vedula GS. In vitro anti-inflammatory and cytotoxicity studies of two mangrove associated lichens, Dirinaria consimilis and Ramalina leiodea extracts. Hygeia J Drugs Med 2018;10(1):16-26.

39. Tatipamula VB, Vedula GS. Anti-inflammatory properties of Dirinaria consimilis extracts in albino rats. J Biomed Sci 2017;4(1):3-8.

40. Tatipamula VB, Vedula GS. Fibrinolytic, Anti-Inflammatory and Cytotoxic Potentialities of Extracts and Chemical Constituents of Manglicolous Lichen, Graphis ajarekarii Patw. \& CR Kulk. Nat Prod J 2020;10(1):87-93. 